\title{
A GLOBAL EXTREMUM PRINCIPLE FOR THE ANALYSIS OF SOLIDS COMPOSED OF SOFTENING MATERIAL
}

\author{
J. E. TAYLOR \\ Department of Aerospace Engineering, College of Engineering, University of Michigan, \\ Ann Arbor, MI 48109, U.S.A. \\ (Received 5 May 1992; in revised form 12 January 1993)
}

\begin{abstract}
An extremum principle is presented covering problems in solid mechanics equilibrium analysis for piecewise linear softening materials. Problems formulated according to this principle are expressed in a mixed "stress and deformation" form. The mechanics interpretation is limited according to linear deformation kinematics. More specialized models, such as an extremum principle in mixed form for linearly elastic materials, an equivalent to the minimum complementary energy principle, and a statement of a bound theorem of Limit Analysis are identified as special cases within the general formulation. Numerical results are presented for two examples of one-dimensional structures made of inhomogeneous, softening material. The evolution of material degradation is demonstrated via a set of solutions obtained for inereasing load. Each solution of the set is produced from a single application of a general purpose computer program for constrained nonlinear programming problems, operating on a finite element interpretation of the nonlinear continuum.
\end{abstract}

\section{INTRODUCTION}

The purpose of this paper is to present a global formulation of equilibrium problems in solid mechanics for systems made of softening material. The formulation is expressed in a way to accommodate arbitrarily inhomogeneous, anisotropic materials. Local "state" is represented in terms of a mixed "stress and deformation" measure. Also, material properties enter the formulation partly in implicit form, through a combination of local and global constraints. By virtue of these several features, a substantial flexibility in the representation of constitutive properties is available in the model (how this is accomplished explicitly becomes clear with the presentation of the problem statement given below). The formulation itself comprises an extremum principle, where a load factor is to be maximized w.r.t. the set of stress and deformation fields. As a result, the sometimes most challenging issues related to existence and uniqueness are in the present circumstances moot, i.e. established results from nonlinear optimization analysis of convex problems fully support a mathematical interpretation of the formulation. Also, making the association (in the usual way) between convexity of the global problem statement and mechanical stability, a category of problems can be identified for which, independent of the specific form of material softening or degradation, the extremum character and thus the stability is preserved indefinitely with increasing load.

The model presented here provides a convenience for the treatment of mechanics problems that require for their interpretation the representation of a set of separate fields, where each field is associated with a separate interval of the region of the solid. Of course problems in the analysis of composites, where the separate fields are identified nominally with the physical structure of constituent materials, belong to this category. Problems involving material degradation, for example where an originally homogeneous medium is rendered through the evolution of structural response into a set of regions each with distinctive constitutive character (e.g. elasto-plasticity), have this property as well. Also, availability of the extremum problem formulation described below is advantageous with respect to the treatment of many of the issues arising routinely in analysis and in the construction of models for computational purposes. Considerations in the treatment of convergence and error analysis, the construction of algorithms, modelling of mechanisms of material degradation, and so on, identify examples of the cited "routinely arising issues". In addition, the present formulation makes it possible to obtain computational solutions 
for structures made of softening materials in a way that circumvents the need to use incremental modelling (this is explained in Section 3 on Computational Results).

The material furnished in this paper is comprised of a presentation of the model for the general problem formulation, a demonstration to show the several specific and/or familiar extremum models imbedded within the general model (complementary energy principle, theorem from limit analysis, etc.), a discussion and interpretation of necessary conditions for the general problem statement, and a sample of results from implementations for the computational treatment of 1-D systems made with inhomogeneous softening material.

\section{PROBLEM FORMULATION}

In the usual paradigm for classical variational modelling of equilibrium problems in elasto-mechanics, mixed formulations amount to stationarity principles, associated in general with saddle-point problems in the stress and deformation variables. This is the case for all the various forms of expression of the mixed principles [see e.g. Washizu (1982)], each of which in effect has one or more equilibrium, constitutive or compatibility conditions imposed as constraints on the functional of one or the other of the classical extremum principles. It is also characteristic in the familiar forms of variational formulation for such problems that the constitutive character of the structural material is represented in explicit form.

The variational formulation described here is distinctive relative to these features, i.e. constitutive character enters the problem statement partly in implicit form (a feasible set in stress-strain space is identified in the problem formulation), and as described in the introduction, the problem statement comprises a global formulation for a mixed model as an extremum problem rather than as a "stationary principle". Also, in the construction of the problem formulation total stress is represented as a superposition of elements from independent sets of admissible fields. Such constructions have precedent in the field of mixture theory and in certain models used in rheology [see e.g. Atkin and Craine (1976)]. The extremum problem reflects maximization of a load factor, where all loads vary in proportion to the load factor (viz. proportional loading), and the requirements of mechanics modelling are introduced as constraints. This latter feature in effect provides an immediate convenience for the relatively broader interpretation of the various attributes of the mechanics that go into the overall definition of an equilibrium field problem. Thus whilc the form of the resulting problem statement is longer and appears to be relatively obtuse, the formulation itself is in fact demonstrably broader and more convenient w.r.t. the business of expressing such problems in general.

As a starting point in the construction of the subject extremum principle, note that the general problem formulation is expressed in terms of two sets of fields, the set $\Sigma$ of at least piecewise differentiable fields (stress constituents) $\sigma^{\gamma} ; \gamma=1,2, \ldots, N_{c}$, and a set $K$ of (admissible displacements) fields $\mathbf{u}$, where for the purposes of the present exposition the $\mathbf{u}$ are continuous and piecewise twice differentiable. (For simplicity, the exposition is described in this limited but relatively simple mathematical setting.) Total stress $\tau$ in the composite medium is expressed as a sum of independent elements from $\Sigma$ plus a stress evaluated on the basis of an element from set $u$, i.e.

$$
\tau_{i j}=\sum_{\gamma=1}^{N_{\mathrm{c}}} \sigma_{i j}^{\gamma}+L_{i j k l} u_{k, l}
$$

The constituent fields $\sigma^{\gamma}$ and $\mathbf{u}$ are at this point arbitrary elements within their respective sets. The constitutive tensor $L_{i j k l}$ interprets the constituent $L_{i j k l} u_{k, l}$ within total stress $\tau$ of (1) in terms of strains $\mathbf{e}(\mathbf{u})$ linear in the gradient of $\mathbf{u}$, for an arbitrarily inhomogeneous, linearly elastic material. 
For convenience in the presentation to follow, the "equilibrium field problem" is expressed in a form consistent with simple boundary conditions. Thus, the total stress $\tau$ of (1) must satisfy:

$$
\begin{array}{ll}
\tau_{i j, j}+\alpha X_{i}=0 & \text { in } \Omega, \\
\tau_{i j} n_{j}-\alpha t_{i}=0 & \text { on } \Gamma_{\mathrm{t}},
\end{array}
$$

and the boundary conditions are summarized with the additional requirement $\mathbf{u}-\mathbf{g}=0$ on $\Gamma_{\mathrm{u}}$ with $\mathrm{g}$ specified, and $\Gamma_{\mathrm{t}}+\Gamma_{\mathrm{u}}=\Gamma$. As indicated above, the representation which has body forces and boundary tractions modified uniformly by the load factor $\alpha$ provides for proportional loading. Also it is supposed that the individual constituent fields $\sigma^{\gamma}$ are constrained (limited) according to some measure of their magnitude. The substance of the results described in this paper does not depend on the particular choice of the measure. For the sake of simplicity, the constraints on stress are expressed for the development that follows in the form of simple "yield conditions". In other words, the fields are required to satisfy

$$
f^{\gamma}\left(\boldsymbol{\sigma}^{\gamma}\right)-a_{\gamma}^{2} \leqslant 0 \quad \gamma=1,2, \ldots, N_{\mathrm{c}}
$$

where functions $f^{\gamma}$ are concave and piecewise smooth in $\sigma^{\gamma}$.

As indicated by the notation in (3), values $a_{\gamma}^{2}$ ("yield limits") and the forms $f^{\gamma}$ are also specified individually for the separate stress fields $\sigma^{\gamma}$. These features, as well as whatever additional detail is necessary to end up with a proper interpretation of the continuum mechanics problem for the composite, show up as constraints in the statement of the extremum problem formulation to be given next.

Note once again that with respect to their rolc in the variational modelling, the stress fields $\sigma^{\nu}$ and "displacement" $u$ are treated as independent. With this in mind, the proposed extremum principle for the (mixed) system is stated :

Maximize load factor $\alpha$ with respect to the fields $\boldsymbol{\sigma}^{\gamma}$ and $\mathbf{u}$ and the scalar $\alpha$ itself, within constraints of reflect (1)-(3) as well as a limit on what amounts to a measure of energy associated with the two fields.

In other words, taking into account conditions (1)-(3), the equilibrium problem of a body formed out of a general anisotropic elastic/softening material is expressed as :

$[\mathrm{P}]$

$$
\max _{\substack{\alpha \varepsilon \Re 1 \\ \sigma^{\gamma} \in \Sigma \\ \mathbf{u} \in K}} \alpha
$$

subject to:

$$
\begin{gathered}
\left(\Sigma_{\gamma} \sigma_{i j}^{\gamma}+L_{i j k l} u_{k, l}\right), j+\alpha X_{i}=0 \text { in } \Omega, \\
\left(\Sigma_{\gamma} \sigma_{i j}^{\gamma}+L_{i j k l} u_{k, l}\right) n_{j}-\alpha t_{i}=0 \quad \text { on } \Gamma_{\mathrm{l}}, \\
f^{\gamma}\left(\boldsymbol{\sigma}^{\gamma}\right)-a_{\gamma}^{2} \leqslant 0 \text { in } \Omega, \\
\Sigma_{\gamma}\left(\int_{\Omega} U_{\mathrm{c}}\left(\sigma^{\gamma}\right) \mathrm{d} V\right)+\int_{\Omega} U(\mathbf{u}) \mathrm{d} V-E \leqslant 0 .
\end{gathered}
$$

Here loads $X_{i}$ and $t_{i}$, constitutive tensor $\mathbf{L}$, "stress limits" $a_{\gamma}$, and "energy bound" $E$ are the data. According to constraint (C3), the solution fields to problem [P] are bounded in their combined energy norm [a similar energy measure appeared in the work of Bendsøe and Sokolowski (1988)]; $U$ and $U_{c}$ represent specific strain energy and specific complementary strain energy, respectively (for the linear strain model) : 


$$
U=\frac{1}{2} L_{i j k l} \varepsilon_{i j} \varepsilon_{k l}, \quad U_{\mathrm{c}}=\frac{1}{2} C_{i j k l} \sigma_{i j} \sigma_{k l}
$$

Properties $L_{i j k l}$ are distinct from $C_{i j k i}$ and are independent of strain state. Note that constraint $(\mathrm{C} 2)$ of problem $[\mathrm{P}]$ comprises an implicit bound on energy $U_{*}$ independent of (C3).

Problem $[\mathrm{P}]$ describes a convex problem within the framework of generalized nonlinear programming analysis [see e.g. Clarke (1983)]. The convexity property assures that a solution identified with the set of necessary conditions from this analysis is unique. It is possible through a comparison of the necessary conditions with the requirements of mechanics to confirm that the result does in fact comprise the solution for equilibrium analysis of continua comprised of the designated form of nonlinear materials. The following additional considerations related to the formulation are noted :

- Having the construction (1) for total stress incorporated within [P] provides for a broad capability in the modelling of material properties. This follows from the feature that the properties of each constituent $\sigma$ " (i.e. the individual moduli $C_{i j k l}$ as well as "strength limits" $f "-\left(a^{2}\right)<0$ ) may be specified separately. so that the total stress $\tau$ may be tailored component by component.

- The formulation $[\mathrm{P}]$ is applicable for arbitrary load state, so long as material tensors $L_{i \text { ik }}$ and $C_{i j k l}$ of the total stress meet the usual thermodynamic requirements for at real material. In other words, within these requirements for constitutive properties, equilibrium of the system is unconditionally stable. Thus it is always possible with this model to find equilibrium solutions over a load regime that carries the solid through its full evolution of material softening (local degradation).

With the imposition of $\left[L_{i j k l}=0 \rightarrow U=0\right]$, problem [P] reduces to the form:

$$
\begin{gathered}
\max \alpha \\
\operatorname{mat} \\
\sigma: \Sigma
\end{gathered}
$$

subject to :

$$
\begin{aligned}
\sum_{i=1}^{N_{i}} \sigma_{i, j}+\alpha X_{i} & =0 \quad \text { in } \Omega, \\
\sum_{i=1}^{N_{c}} \sigma_{i j} n_{j}-\alpha t_{i} & =0 \quad \text { on } I_{i}, \\
f(\sigma)-a_{j} & \leqslant 0 \quad \text { in } \Omega \quad i=1,2, \ldots, N_{i}, \\
\Sigma_{i} \int_{\Omega} U_{i}(\sigma) d V-E & \leqslant 0 .
\end{aligned}
$$

The set $\Sigma$ of stress fields $\sigma^{\prime \prime}$ for this problem is the same as the one defined above. Problem $[\mathrm{P} 1]$ is a slightly generalized form of the unified statement for "idealized elastoplasticity" reported earlier [Taylor (1989), Ben-Tal and Taylor (1991)]. That is. [P1] interpeted for a single stress field (i.e. $N_{\mathrm{c}}=1$ ) corresponds to "deformation plasticity theory" for the elastic/perfectly plastic idealization. Hlavacek et al. reported on a different "alternative formulation" in their recent paper [Hlavacek et al. (1992), also see Ciarlet (1991), and Comi et al. (1992)]. For $N_{\mathrm{c}} \geqslant 2$ it represents a piecewise-linear softening material with an ultimate capacity corresponding again to an idealized plasticity-like limit. This constitutive simulation matches in form the stress-strain character of the "Besseling material" (Besseling, 1984). As a comparison, the net constitutive character incorporated in formulation [P] is similar, but it provides via the term $L_{i j k l} u_{k, l}$ for a suslained elastic reserve capacity, rather than the "ideally plastic" limit of the Besseling material.

To continue the interpretation, the classical model identified with the upper bound theorem of "Limit Analysis" may be recovered in turn from [P1]. This result, namely 


$$
\max _{\substack{\alpha \in \Re^{1} \\ \sigma \in \Sigma}} \alpha
$$

subject to :

$$
\begin{array}{ll}
\sigma_{i j, j}-\alpha X_{i}=0 & \text { in } \Omega, \\
\sigma_{i j} n_{j}-\alpha t_{i}=0 & \text { in } \Gamma_{\mathrm{t}}, \\
f(\sigma)-a^{2} \leqslant 0 & \text { in } \Omega,
\end{array}
$$

follows from [P1] for $N_{\mathrm{c}}=1$ and the condition that (data value) $E$ is sufficiently large relative to the specified "yield limit" value (the energy constraint becomes superfluous).

It is possible also to recover from [P1] a statement equivalent to the classical "Minimum Complementary Potential Energy" principle. This result, obtained for the converse limits on the constraint bound values in [P1], namely for limits $a_{\gamma} ; \gamma=1,2, \ldots, N_{\mathrm{c}}$ sufficiently large relative to the energy constraint bound $E$ (the "yield limits" are rendered inactive throughout the domain of the body), is

$$
\max _{\substack{\alpha \in \mathfrak{P}^{1} \\ \sigma \in \Sigma}} \alpha
$$

subject to:

$$
\begin{aligned}
\Sigma_{\gamma} \sigma_{i j, j}^{\gamma}-\alpha X_{i}=0 & \text { in } \Omega, \\
\Sigma_{\gamma} \sigma_{i j}^{\gamma} n_{j}-\alpha t_{i}=0 & \text { on } \Gamma_{\mathrm{t}}, \\
\Sigma_{\gamma} \int_{\Omega} U_{\mathrm{c}}\left(\boldsymbol{\sigma}^{\gamma}\right) \mathrm{d} V-E \leqslant 0 . &
\end{aligned}
$$

Note that the "equivalence" may be confirmed (to the level of a stationary principle) through an examination of the necessary conditions of [P3]. Also, the superposition of fields $\boldsymbol{\sigma}^{\gamma}$ in [P3] is redundant, i.e. the model simply represents elastostastics for a linearly elastic system with "material stiffness" equal to that of the combined constituent fields working in parallel.

Two additional points of interest are cited in connection with the variational problem statement. First, it may be more convenient in certain circumstances to work with the original (i.e. most general) formulation expressed in the alternative form:

$$
\min _{\substack{\alpha \in \Re^{1} \\ \sigma \sigma \Sigma \\ u \in K}}\left\{\int_{\Omega}\left[\Sigma_{\gamma} U_{\mathrm{c}}\left(\sigma^{\gamma}\right)+U\right] \mathrm{d} V\right\}
$$

subject to :

(Equilibrium equation and stress boundary condition as in [P])

$$
\begin{gathered}
f^{\gamma}\left(\sigma^{\gamma}\right)-a_{\gamma}^{2} \leqslant 0 \quad \text { in } \Omega, \quad \gamma=1,2, \ldots, N_{\mathrm{c}}, \\
\alpha-\alpha \leqslant 0 .
\end{gathered}
$$

Version $\left[\mathrm{P}^{\prime}\right]$, where the argument of the former energy constraint is to be minimized while the load $\alpha$ is now constraincd from bclow, is equivalent to [P]. Observe that the load constraint is active at the solution, and so the value $\underline{\alpha}$ represents in effect a specified load (of course this kind of alternative formulation is also available for the various problems [P1]-[P3] discussed above). Given the convexity of the two problems, the equivalence of $[\mathrm{P}]$ and $\left[\mathrm{P}^{\prime}\right]$ can be verified simply on the basis of a comparison of their necessary conditions.

Lastly, it is noted that for the limit $\left\{a_{\gamma} \rightarrow 0\right.$; all $\left.\gamma\right\}$ the original problem [P] reduces to the statement : 
subject to :

$$
\begin{aligned}
\left(L_{i j k l} u_{j, k}\right)_{, l}+\alpha X_{i}=0 & \text { in } \Omega, \\
L_{i j k l} u_{j, k} n_{l}-\alpha t_{i}=0 & \text { on } \Gamma_{1}, \\
\int_{\Omega} U[\boldsymbol{\varepsilon}(\mathbf{u})] \mathrm{d} V-E & \leqslant 0 .
\end{aligned}
$$

The energy constraint is active at the solution, and so this problem is identified with the relatively uninteresting result that the equilibrium field that maximizes load maximizes total strain energy.

In summary, problem [P] (and its equivalent $\left[\mathrm{P}^{\prime}\right]$ ) comprises an extremum principle for the global response of a system made of a nonuniform elastic/softening material. Solutions generated for a set of increasing data values of energy bound $E$ collectively provide information on the evolution of stress and deformation fields with increasing load. The formulation as stated is a "mixed" (i.e. stress and displacement) form. Note that for the case $N_{\mathrm{c}}=1$ and data value $a_{\gamma}$ sufficiently large so that constraint (C2) is inactive and with $L_{i j k l}=\left(C_{i j k l}\right)^{-1}$, formulation [P] (or [ $\left.\mathrm{P}^{\prime}\right]$ ) becomes simply the statement of a "mixed principle" for a linearly elastic material.

\section{NECESSARY CONDITIONS ; SUFFICIENCY}

Formulation $[\mathrm{P}]$ has been presented as a characterization of equilibrium mechanics for an inhomogeneous, general form of anisotropic, softening material. The constitutive character of the material is represented in the model via the stiffness tensor $L_{i j k l}$, the set of compliance tensors $C_{i j k l}^{\gamma}$, and the set of "stress limit conditions" $f^{\nu}\left(\sigma^{\gamma}\right)-a_{i}^{2} \leqslant 0$. The problem statement is supportable within the context of standard results in the mathematical modelling of nonlinear, nonsmooth optimization problems [see e.g. Clarke (1983)]. A moderately detailed exposition of the necessary conditions associated with $[\mathrm{P}]$ is presented next, with the idea that an examination of these conditions provides for an interpretation of the model on grounds that are least in part closer to more familiar descriptions in solid mechanics.

Stationarity in problem [P] with respect to variation of the scalar $\alpha$ (load factor) requires $\left(\lambda_{\mathrm{e} i}\right.$ and $\lambda_{\mathrm{b} i}$ symbolize multipliers associated with the equilibrium field equations and stress boundary conditions respectively):

$$
-1+\int_{\Omega} \lambda_{\mathrm{e} i} X_{i} \mathrm{~d} V-\int_{\Gamma_{\mathrm{r}}} \lambda_{\mathrm{b} i} t_{i} \mathrm{~d} A=0
$$

This comprises a load weighted normalization of the multiplier functions $\lambda_{e i}$ and $\lambda_{b i}$. Necessary conditions associated with variation among constituent stress fields $\sigma^{\gamma}$ may be expressed :

$$
\begin{aligned}
\left(\lambda_{\mathrm{e} i}+\hat{\lambda}_{\mathrm{b} i}\right) n_{j}=0 & \text { on } \Gamma_{\mathrm{t}}, \\
\left(\lambda_{\mathrm{c} i}+\lambda_{\mathrm{bi} i}\right) n_{j}=0 & \text { on } \Gamma_{\mathrm{t}}, \\
\lambda_{\mathrm{e} i} n_{j}=0 & \text { on } \Gamma-\Gamma_{\mathrm{t}}, \\
-\left(\lambda_{\mathrm{e}, i}+\lambda_{\mathrm{e}, i, i}\right) / 2+\mu^{\beta} \frac{\partial f^{\beta}}{\partial \sigma_{i, j}^{\beta}}+\Lambda C_{i j k l}^{\beta} \sigma_{k l}^{\beta}=0 & \text { in } \Omega, \quad \beta=1,2, \ldots, N_{\mathrm{c}},
\end{aligned}
$$

$\mu^{\beta}$ and $\Lambda$ represent the multipliers on constraints (C2) and (C3) respectively. Stationarity w.r.t. variation of the displacement field calls for the solution of $[\mathrm{P}]$ to satisfy: 


$$
\begin{aligned}
L_{i j k l}\left(-\lambda_{\mathrm{e} i, j}+\Lambda \varepsilon_{i j}\right)=0 & \text { in } \Omega, \\
L_{i j k l}\left(\lambda_{\mathrm{ei}}+\lambda_{\mathrm{bi}}\right) n_{j}=0 & \text { on } \Gamma_{\mathrm{t}}, \\
L_{i j k l} \lambda_{\mathrm{ei}} n_{j}=0 & \text { on } \Gamma-\Gamma_{\mathrm{t}} .
\end{aligned}
$$

The complementarity condition for the material degradation constraint (C2) is stated:

$$
\mu^{\beta}\left[f^{\beta}\left(\sigma^{\beta}\right)-a_{\beta}^{2}\right]=0 \quad \text { in } \Omega, \quad \beta=1,2, \ldots, N_{\mathrm{c}} .
$$

Also the condition

$$
\mu^{\beta} \geqslant 0 \quad \text { in } \Omega, \quad \beta=1,2, \ldots, N_{\mathrm{c}}
$$

must be met at the solution.

Toward an interpretation of this (partial) list of necessary conditions; note from the boundary equations of (5) and (6) that $\lambda_{b i}=-\lambda_{e i}$ on $\Gamma$. In fact, by the combined equations (6)

$$
\tilde{\lambda}_{\mathrm{e} i}=u_{i} \text { in } \Omega
$$

$\left(\tilde{\lambda}_{\mathrm{e} i}=\lambda_{\mathrm{e} i} / \Lambda\right.$ and $\tilde{\mu}^{\beta}=\mu^{\beta} / \Lambda$ are introduced for convenience and w.o.l.o.g.). Accordingly, the field equation (5) can be written :

$$
\varepsilon_{i j}=C_{i j k l}^{\beta} \sigma_{k l}^{\beta}+\tilde{\mu}^{\beta} \frac{\partial f^{\beta}}{\partial \sigma_{i j}^{\beta}} \text { in } \Omega, \quad \beta=1,2, \ldots, N_{\mathrm{c}} .
$$

The set of intervals in $\Omega$ where for the $\beta$ th constituent $f^{\beta}\left(\sigma^{\beta}\right)<a_{\beta}^{2}$ is identified by $\Omega_{\mathrm{E} \beta}$. According to (7), $\mu^{\beta}=0$ in $\Omega_{\mathrm{E} \beta}$ and so from (9):

$$
\varepsilon_{i j}=C_{i j k l}^{\beta} \sigma_{k l}^{\beta} \text { in } \Omega_{\mathrm{E} \beta}
$$

In other words, $\Omega_{\mathrm{E} \beta}$ are these intervals in which the $\beta$ th constituent behaviour is unsoftened, and (10) reflects the requirement of compatibility among these constituent fields and the field $\boldsymbol{\varepsilon}(\mathbf{u})$. In the alternative situation, i.e. where $\mu^{\beta} \geqslant 0 ; f^{\beta}(\hat{\boldsymbol{\sigma}} \beta)=a_{\beta}^{2}$ (notation $(\hat{\boldsymbol{\sigma}} \beta)$ identifies stress values on the "limiting surface"), from (9)

$$
\varepsilon_{i j}=C_{i j k l}^{\beta} \hat{\sigma}_{k l}^{\beta}+\mu^{\beta} \frac{\partial f^{\beta}}{\partial \sigma_{i j}} \text { in }\left(\Omega-\Omega_{\mathrm{E} \beta}\right)
$$

Here the second term on the right-hand side of (11) reflects the difference between total strain $\varepsilon_{i j}$ and the measure of strain corresponding to $\hat{\sigma}_{i j}$ on the "limit surface" (this quantity corresponds to what is identified in the appropriate context as the "plastic strain"; here it simply reflects a relaxation of the above-mentioned compatibility).

Since in problem $[\mathrm{P}]$ (as in equivalent $\left[\mathrm{P}^{\prime}\right]$ ) constraint $(\mathrm{Cl})$ is linear and the (energylike) (C3) is quadratic, convexity of the problem depends only on the requirement that functions $f^{\prime}\left(\sigma^{\gamma}\right)$ in constraint (C2) are concave. Thus it may be appreciated that the convexity property prevails for the full range of models of material degradation (softening) covered within this simple requirement on the form of (C2). As noted in Section 1, for such convex problems the set of necessary conditions for [P] are also sufficient (sufficiency for this problem can also be verified easily via classical arguments). Note also that the system is mechanically stable (unconditionally with respect to load so long as $\varepsilon(u)$ is not identically zero, and the requirements of Section 1 on constitutive tensors are met). Given these properties, the variational formulation may be directly useful as a basis for the development of computational means, for example, or to establish bounds on the overall properties of 
composites, and so on. These and other issues related to applications are discussed briefly below.

\section{APPLICATIONS FOR COMPUTATIONAL RESULTS}

Procedures for the numerical solution of constitutively nonlinear problems generally are based on an interpretation that is incremental with respect to the load/deformation process, with special means (updating or corrector steps) incorporated to limit cumulative error. On the other hand, for problems where the present extremum formulation is applicable it is possible to obtain numerical solutions using computational means for direct minimization. This alternative to incremental methods is applied here, i.e. a rendering of formulation [P] into discrete (computational) form is used to obtain sets of solutions for two rudimentary example problems in one-dimensional continua. Given the interpretation into discrete form, these solutions are produced using a software package (EMP by Klaus Schittkowski, University Bayreuth, D 8580 Bayreuth) created for the treatment of constrained problems in nonlinear optimization. Examples were solved using a commercial program (Abaqus) based on a conventional incremental model as well, in order to provide a comparison of results.

A computer program was written in order to obtain solutions for problems of an axially loaded continuum bar fixed at its ends. The program reflects an interpretation of problem formulation $[\mathrm{P}]$ via a finite element model for the one-dimensional structure made up of arbitrarily inhomogeneous, softening materials. The two specific example structures to be analysed are shown schematically in Fig. 1. The lowest admissible order of element

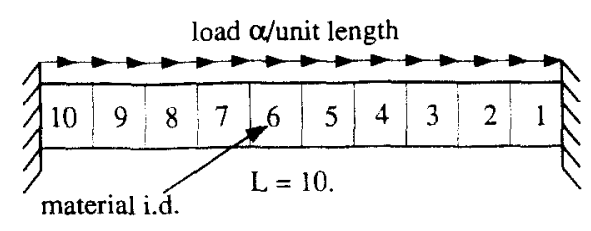

Loads, Supports, and Element Numbers - First Example Problem

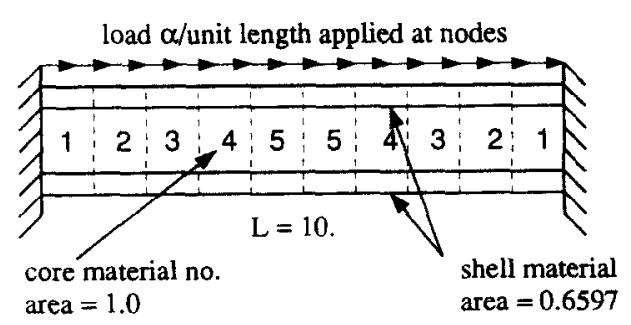

Loads, Supports, and Element Numbers - Second Example Problem

Fig. 1. Schematic diagrams of the structures and loads for the two example problems. 

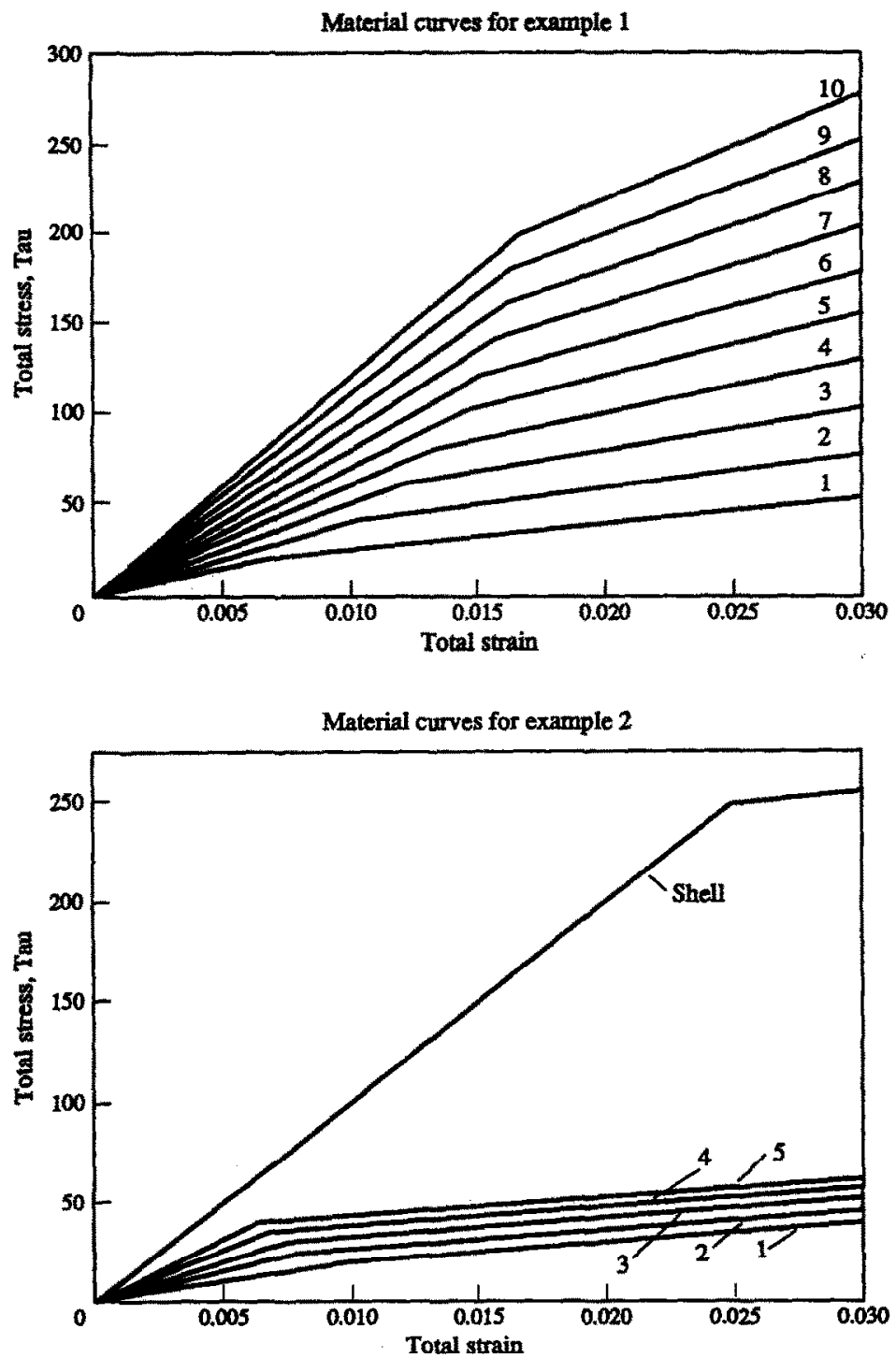

Fig. 2. Total stress $\tau$ versus strain $\varepsilon$ for each element of the model for Example l.

interpolation functions consistent with the (mixed) model were used, namely "constant stress" and "linear displacement" elements.

In order to show an application to the analysis for a specifically inhomogeneous structure, a 10-element model was set up to simulate a situation where both stiffness and "onset of softening" vary over the length of the structure. The element constituent properties for this case are given in Fig. 2. Results for an analysis of the bar under uniformly distributed load, reflecting the evolution of response under increasing load, are given in the form of total stresses in Fig. 3, and displacements in Fig. 4. The change in character of the "stress distribution" with increasing load simply reflects the effect of progressive material degradation in this example. For low load level and before softening occurs, the interval $\Omega_{E \beta}$ is identified with the entire length of the bar. Softening progresses from the leftmost element uniformly toward the right-hand end as the load is increased, so the unsoftened interval $\Omega_{E \beta}$ is identified with a shrinking portion of the bar adjacent to the right support.

The structure of the second example, still one-dimensional, is made up of two parallel layers each with distinct properties. The load is applied to one layer and so the example provides a possibility to observe how loads (stresses) diffuse between the layers. Again, the change in the pattern of this redistribution of stresses as a consequence of the material 


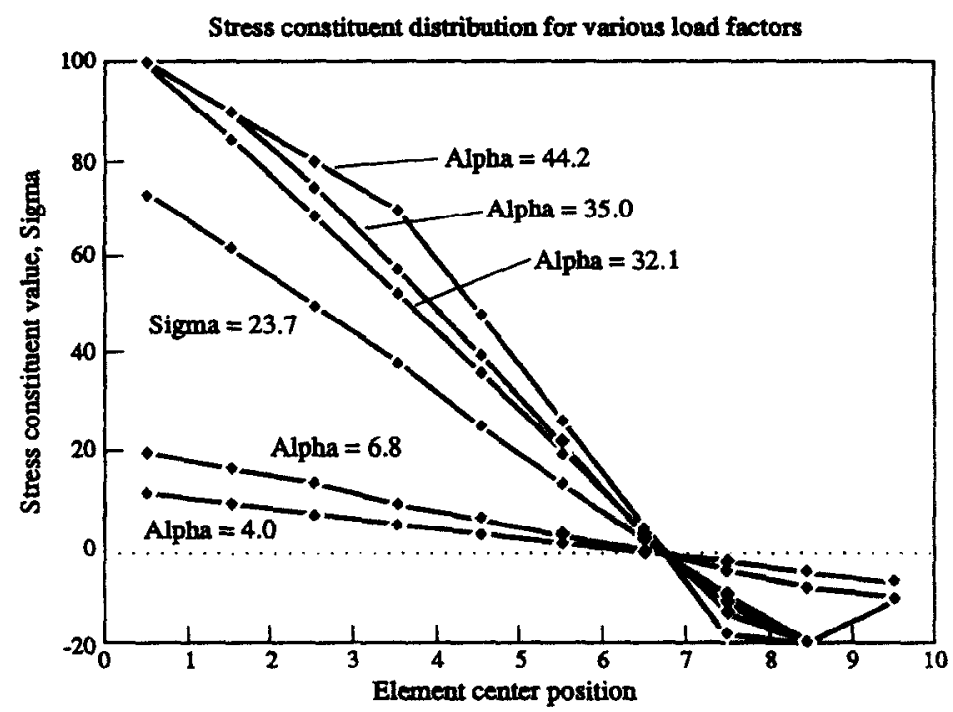

Fig. 3. Stress distributions for various loads (Example 1).

softening can be observed ; this is shown in Fig. 5, where total stresses in the "core material" and the "shell material" are plotted. A set of results showing the evolution of deformation response for this example is given in Fig. 6.

An application was made to the commercial program Abaqus to obtain a comparison solution for the load $\alpha=44.2$ in the first example problem; the evolution of material softening is well devloped at this relatively high level of loading. Using a 10-element model here to match the one used with the extremum formulation, the results as represented by total stresses and displacements evaluated to four significant figures were indistinguishable. Results for the second example loaded to a level $\alpha=49.7$ are compared in Table 1 . Here the worst discrepancy between results obtained by direct minimization and those produced using Abaqus is of the order of $1.1 \%$ For this result the loading process was interpreted via 65 increments within the workings of Abaqus.

It is not the intention with the presentation of the examples in this section to argue that the approach for numerical solution via direct minimization is in any sense better

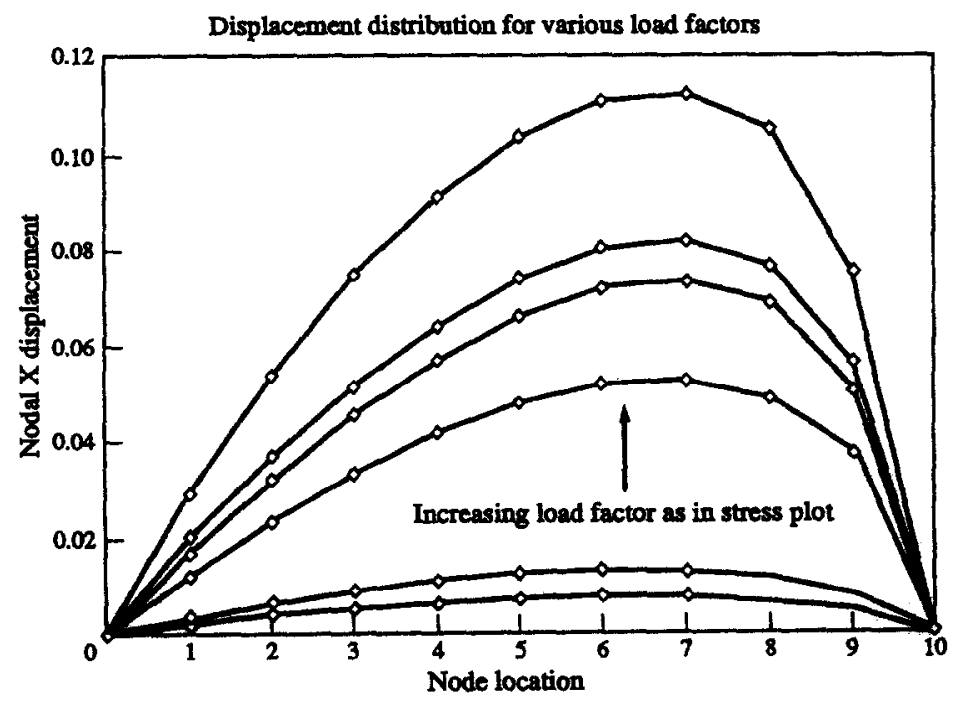

Fig. 4. Displacements at points along the bar for various loads (Example 1). 

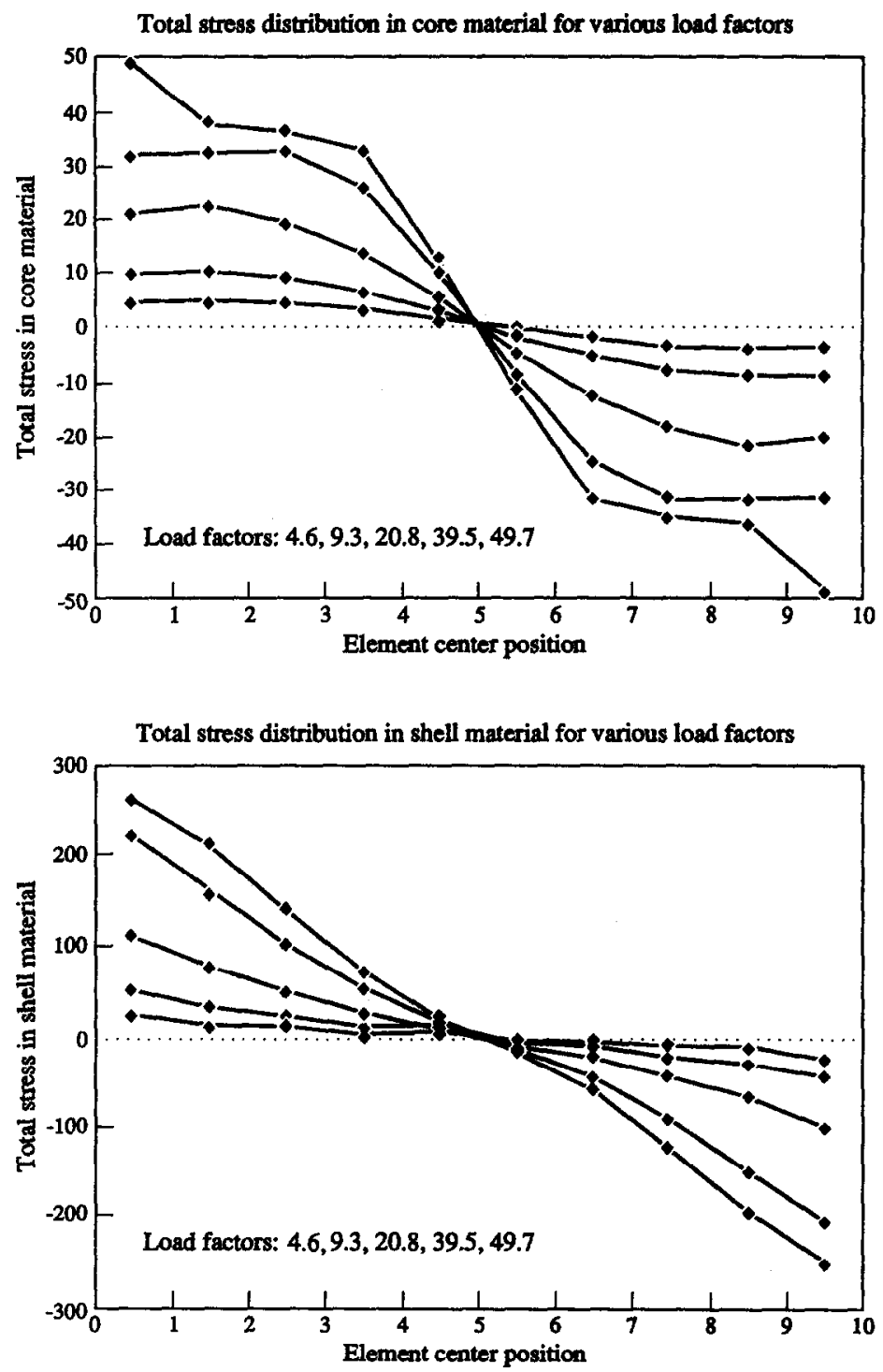

Fig. 5. Stresses in "shell" and "core" materials for the two layered bar of Example 2.

than conventional approaches. Judgement on the relative merit of computational means developed on the basis of the extremum problem formulation for the subject class of nonlinear problems cannot be made without first having an implementation done and a thorough survey of applications made for two- and three-dimensional problems.

\section{SUMMARY}

One should expect that certain known results available in structural analysis based on linearly elastic material behavior may be extended in a straightforward way to accommodate softening materials, using the formulation described in this paper. The usefulness of being able to identify with an extremum principle for the purpose of development of computational models is cited as an example. It is reasonable to suppose that the sort of modelling represented in Teply and Reddy (1991), where a particular form (Aboudi's model) of a two-material composite is interpreted directly into a finite element form, is applicable for the composite of nonlinear materials as well, without significant change. Also the methods of analysis used to predict bounds on the effective properties of composites [e.g. Milton and Kohn (1988)] should also apply for the composite with softening constituent 


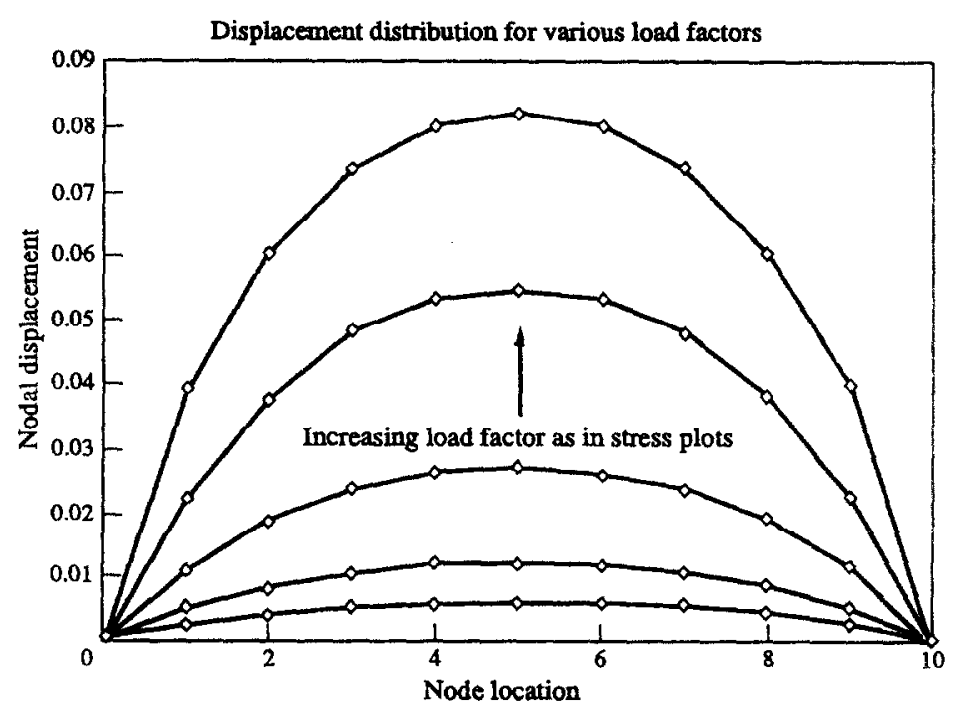

Fig. 6. Displacements at points along the bar for various loads (Example 2 )

materials, within the limitation to "proportional loading". It is interesting as well to speculate on what conveniences might be available in connection with the modelling discussed in Talbot and Willis (1991) for constitutively nonlinear composites, or in relation to the subject of damage mechanics at large. With respect to the latter of these areas, the flexibility to express modelling for local damage in terms of both stress and deformation variables- which convenience is afforded by virtue of having the equilibrium mechanics represented via a "mixed model"- is noteworthy. At the same time, it is important to bear in mind that the formulation of this paper as it stands is not proposed for use in situations where stress/strain reversal occurs.

As noted in the introduction, the representation in formulations $[\mathrm{P}]$ and $\left[\mathrm{P}^{\prime}\right]$ of total stress in terms of a superposition of independently controlled constituent fields provides for considerable flexibility in the simulation of properties for softening materials. The procedure by which such simulation is accomplished can be formalized without difficulty. In fact the global formulation accommodates a generalized form of superposition construction, i.e. a form where the constituent fields are arbitrary but derivable from potentials.

In another area, the extremum principle $[P]$ provides a most convenient basis for the treatment of problems in the optimal design of composites composed of (possibly) softening constituents [this kind of application is exemplified for discrete structures in Taylor and Logo (1992)].

Table 1 . Comparison of results for load $\alpha=49.7$ in example 1 wo

\begin{tabular}{|c|c|c|c|c|}
\hline \multicolumn{5}{|c|}{ Solution values for total stress } \\
\hline \multirow{2}{*}{$\begin{array}{l}\text { Element } \\
\text { position }\end{array}$} & \multicolumn{2}{|c|}{$\begin{array}{l}\text { By direct minimization } \\
\text { formulation }[\mathrm{P}]\end{array}$} & \multicolumn{2}{|c|}{$\begin{array}{l}\text { Produced using the program } \\
\text { Abaqus }\end{array}$} \\
\hline & Core material & Shell material & Core material & Shell material \\
\hline 0.5 & 49.29 & 264.3 & 49.29 & 264.3 \\
\hline 1.5 & 37.37 & 207.0 & 37.37 & 207.0 \\
\hline 2.5 & 35.89 & 133.9 & 35.89 & 133.9 \\
\hline 3.5 & 32.16 & 64.26 & 32.14 & 64.28 \\
\hline 4.5 & 11.97 & 19.53 & 11.84 & 19.73 \\
\hline 5.5 & -11.97 & -19.53 & -11.84 & -19.73 \\
\hline 6.5 & -32.16 & -64.26 & -32.14 & -64.28 \\
\hline 7.5 & -35.89 & -133.9 & -35.89 & -133.9 \\
\hline 8.5 & -37.37 & -207.0 & -37.37 & -207.0 \\
\hline 9.5 & -49.29 & -264.3 &.--49.29 & -264.3 \\
\hline
\end{tabular}


Acknowledgements-Thanks are due to Sheldon Plaxton for the production of the numerical results reported in this paper. Also, the author is indebted to the Mathematical Institute, Danish Technical University for support during his stay in Lyngby, graciously hosted by Martin Bendsøe, in the fall of 1989.

\section{REFERENCES}

Atkin, R. J. and Craine, R. E. (1976). Continuum theory of mixtures: Applications. J. Inst. Math. Appln. 17, 153-207.

Bendsøe, M. P. and Sokolwski, J. (1988). Design sensitivity analysis for elastoplastic analysis problems. Mech. Struc. Mach. 16, 81-102.

Ben-Tal, A. and Taylor, J. E. (1991). A unified model for elastoplastic structural analysis via dual variational principles. Proc. SIAM-Dayton Conf. on Design Theory (Edited by V. Komkov and D. Field) Waverly Press, Easton, MD.

Besseling, J. F. (1984). Models of metal plasticity: Theory and experiment. In Plasticity Today (Edited by A. Sawczuk and G. Bianchi). Elsevier, London, New York.

Ciarlet, P. G. (1991). Basic error estimates for elliptic problems. Finite Element Methods (part I)/Handbook of Numerical Analysis (Edited by P. G. Ciarlet and J. L. Lions). Elsevier Science Publishers B.V./North Holland, Amsterdam).

Clarke, F. H. (1983). Optimization and Nonsmooth Analysis. Wiley, New York.

Comi, C., Maier, G. and Perego, V. (1992). Generalized variable finite element modeling and extremum theorems in stepwise molonomic elastoplasticity with internal variables. Comp. Meth. Appl. Mech. Engng 96, 213-237.

Hlavacek, I., Rosenberg, J., Beagles, A. E. and Whiteman, J. R. (1992). Variational inequality formulation in strain space and finite element solution of an elasto-plastic problem with hardening. Comp. Meth. Appl. Mech. Engng 94, 93-112.

Milton, G. W. and Kohn, R. V. (1988). Variational bounds on the effective moduli of anisotropic composites. J. Mech. Phys. Solids 36(6), 597-629.

Talbot, D. R. S. and Willis, J. R. (1991). The overall behaviour of fiber reinforced composite. In Inelastic Deformation of Composite Materials (Edited by George J. Dvorak), pp. 527-546. Springer, New York.

Taylor, J. E. (1989). Toward a unified model for elastoplastic structural analysis. Mech. Res. Comm. 6(2), $125-131$.

Taylor, J. E. and Logo, J. (1992). Analysis and design of elastic/softening truss structures based on a mixed-form extremum principle. Proc. NATO/FRG Conf. Optimization of Large Structural Systems, 23 Scptcmber-4 October 1991 (Edited by George Rozvany). Kluwer Academic Publishers, Dordrecht (to appear).

Teply, J. L. and Reddy, J. N. (1991). A Unified Formulation of Micromechanics Models of Fiber-Reinforced Composites. In Inelastic. Deformation of Composite Materials (Fdited by George J. Dvorak), pp. 341-372. Springer, New York.

Washizu, K. (1982). Variational Methods in Elasticity and Plasticity (3rd Edn). Pergamon Press, New York. 